Proceedings of Anticancer Research

Research Article

\title{
Clinical Observation of Huagai Pingchuan Decoction Combined with Seretide in the Treatment of Asthma
}

\author{
Rong He
}

Affiliated Hospital of Shandong University of traditional Chinese medicine, Jinan City 250014, Shandong Province, China

Funding: The Sixth Batch of national old TCM experts' academic experience inheritance project

\begin{abstract}
Objective: To study the clinical effect of the Huagai Pingchuan decoction combined with Seretide in the treatment of asthma. Methods: 138 cases of asthma patients in our hospital were separated into the control group and the experimental group. The control group was given Seretide alone, while the experimental group was treated with Huagai Pingchuan decoction combined with Seretide. Results: After treatment, the wheezing symptoms remission time of the experimental group was shorter than control group, the performance of serum inflammatory factors and lung function indexes were better than control group, $\mathrm{P}<0.05$ with significant difference. Conclusion: Huagai Pingchuan decoction combined with Seretide is more effec-tive in the treatment of asthma.
\end{abstract}

Keywords: Huagai Pingchuan decoction; Seretide; Asthma

Publication date: September, 2020

Publication online: 30 September, 2020

*Corresponding author: Rong He, emailhr@163.com

Asthma mainly refers to bronchial asthma, mainly causing by the respiratory system disease participated of multiple cells or cell composition, the occurrence may be related with inheritance, environmental allergens and other factors, asthma patients often showing wheezing repeatedly, shortness of breath, chest tightness, cough and other symptoms, Western medicine still unable to radically cure asthma currently. Most of them applying oxygen inhalation, anti-inflammatory and cough relieving methods. Since asthma is easy to reappear, long-term taking Western medicine may lead to drug resistance, which has a great impact on the prognosis and quality of life of patients ${ }^{[1-2]}$. According to the relevant research, from the perspective of traditional Chinese medicine, the application of traditional Chinese medicine combined with Western medicine can play a significant role in the treatment of asthma. Based on this, this paper selected 138 cases of asthma patients in our hospital from May 2019 to June 2020 to study the therapeutic effect of Huagai Pingchuan decoction combined with Seretide.

\section{Data and methods}

\subsection{Basic data}

From May 2019 to June 2020, the medical staff of our hospital carried out a one-year study. During this period, 138 patients with asthma were treated as the research objects. All the objects had excluded patients with severe liver and kidney disorders and drug allergy, and voluntarily signed the research consent. The patients were separated into the control group and the experimental group by drew lots, with 69 cases in each group. The proportion of male and female in the control group was $38: 31$, and the average was (48.25 \pm 1.52$)$ years old. The shortest course of disease was 1 year, the longest was 9 years, and the average was (5.12 \pm 0.38 ) years; the proportion of male and female in the experimental group was 37:32, the age range was 3763 years, the average age range was $(48.17 \pm 1.48)$ years, and the shortest course of disease was 1 year, the longest was 8 years, with an average of $(5.10 \pm 0.34)$ years. Statistical comparison of the two data groups, $\mathrm{P}>$ 0.05 , no significant difference.

\subsection{Method}

All patients need was given oxygen inhalation, 
expectorant, anti cough, anti infection and other conventional treatment after admission. The control group was treated with western medicine (Seretide provided by GlaxoSmithKline investment company) for inhalation treatment, 1 inhalation each time, 2 times a day, continuous treatment for 2 weeks; the experimental group was treated with Seretide combined with antiasthmatic traditional Chinese medicine, with specific formula: ginseng, Astragalus membranaceus, Atractylodes macrocephala, roasted ephedra, almond, chuanxiong, clam, each in 15 grams, 10 grams of Scutellaria baicalensis, 10 grams of white mustard seed, and 5 grams of roasted licorice were decocted with water. The decoction was taken as one dose, one dose a day, two times in the morning and evening. The treatment lasted for 2 weeks.

\subsection{Observation index}

The remission time of wheezing symptoms in the daytime and at night was statistically observed; the pulmonary function indexes such as FEV1 (first forced expiratory volume), FVC (forced vital capacity) and PEFR (peak respiratory velocity), and serum inflammatory factors such as TNF- $\alpha$ (serum tumor necrosis factor- $\alpha$ ), IL-6 (interleukin-6) and CRP (C-reactive protein) before and after treatment were statistically observed. If the clinical symptoms and lung moist rales have subsided, it can be judged as cured; if the symptoms and lung moist rales have improved significantly, they can be judged as effective; if the above symptoms and signs have no change, it is failed, and the total recovery rate $=($ cured + effective $) /$ total cases $\times 100 \%$.

\subsection{Statistical analysis}

SPSS 23.0 was used to calculate the data, $\mathrm{T}$ and $\mathrm{X} 2$ tests were performed, which were expressed as $(\bar{x} \pm \mathrm{s})$, (n $/ \%)$. If $\mathrm{P}<0.05$, the data were significant different.

\section{Results}

\subsection{Statistical assessment of pulmonary function indicators of patients in the two groups}

According to the data statistics in the table below, there was no significant difference in lung function indexes between the two groups before treatment. After treatment, it was shows that the improvement of lung function indexes in the experimental group was better with significant difference $(\mathrm{P}<0.05)$.

\subsection{Statistical evaluation of serum inflammatory factors index of the two groups}

According to the statistics in Table 2, there was no significant difference in inflammatory factor indexes between the two groups before treatment. After

Table 1. Statistical Evaluation of lung function indexes $(x \pm s)$

\begin{tabular}{ccccccc}
\hline \multirow{2}{*}{ group } & \multicolumn{2}{c}{ FEV1 } & \multicolumn{2}{c}{ FVC } & \multicolumn{2}{c}{ PEFR } \\
\cline { 2 - 7 } & Before treatment & After treatment & Before treatment & After treatment & Before treatment & After treatment \\
\hline Control group & $67.52 \pm 5.41$ & $73.14 \pm 6.57$ & $2.61 \pm 0.47$ & $3.09 \pm 0.41$ & $361.58 \pm 26.24$ & $403.47 \pm 22.19$ \\
Research Group & $67.81 \pm 5.62$ & $77.69 \pm 6.41$ & $2.63 \pm 0.49$ & $3.48 \pm 0.31$ & $360.47 \pm 26.17$ & $433.24 \pm 28.59$ \\
$t$ & 0.3088 & 4.1175 & 0.2446 & 6.3026 & 0.2487 & 6.8398 \\
$P$ & 0.7579 & 0.0001 & 0.8071 & 0.0000 & 0.8039 & 0.0000 \\
\hline
\end{tabular}

Table 2. Statistical evaluation of inflammatory factors

\begin{tabular}{ccccccc}
\hline \multirow{2}{*}{ group } & \multicolumn{2}{c}{ TNF- $\alpha$} & \multicolumn{2}{c}{ IL-6 } & & CRP \\
\cline { 2 - 7 } & Before treatment & After treatment & Before treatment & After treatment & Before treatment & After treatment \\
\hline Control group & $83.52 \pm 11.28$ & $43.58 \pm 9.58$ & $20.14 \pm 4.59$ & $11.98 \pm 3.06$ & $18.59 \pm 5.84$ & $7.81 \pm 2.26$ \\
Research Group & $83.47 \pm 11.17$ & $38.11 \pm 8.74$ & $20.23 \pm 4.71$ & $9.72 \pm 2.84$ & $18.83 \pm 5.26$ & $5.15 \pm 1.99$ \\
$t$ & 0.0261 & 5.4255 & 0.1136 & 4.4967 & 0.2536 & 7.3376 \\
$P$ & 0.9792 & 0.0001 & 0.9097 & 0.0000 & 0.8001 & 0.0000 \\
\hline
\end{tabular}


Table 3. Statistical evaluation of the treatment effect of the two groups (n /\%)

\begin{tabular}{|c|c|c|c|c|c|}
\hline Group & $\mathrm{N}$ (cases) & Cured & Effective & Failed & Total recovery rate \\
\hline Control group & 69 & 21 & 33 & 15 & 78.26 \\
\hline Research Group & 69 & 26 & 37 & 6 & 91.30 \\
\hline $\mathrm{t}$ & & & & & 4.5495 \\
\hline $\mathrm{P}$ & & & & & 0.0329 \\
\hline
\end{tabular}

treatment, the inflammatory factor indexes of the experimental group were better than those of the control group, with significant difference.

\subsection{Statistical evaluation of the remission time of wheezing symptoms of the two groups}

After the application of treatment, the remission time of wheezing symptoms in the day and night of the control group was $(3.81 \pm 1.15)(5.38 \pm 1.74)$ days, and the experimental group was $(3.01 \pm 0.54)(4.51 \pm 0.96)$ days respectively. After comparison, it shows that the remission time of the experimental group was shorter, with significant difference $(\mathrm{t}=5.2305,3.6365, \mathrm{P}<0.05)$.

\subsection{Statistical evaluation of the recovery rate of the two groups}

From statistics analysis, it can be found that the total recovery rate of the experimental group is higher, $\mathrm{P}<0.05$, there is a significant difference between the groups.

\section{Discussion}

Asthma is a very common respiratory disease in clinical study. Patients often have congestion or edema of airway mucosa due to the spasm of bronchial smooth muscle, resulting a lot of inflammatory exudates, causing cough and wheezing. Currently, there is no radical cure for asthma. For western medicine clinical treatment, majority of them are using $\beta 2$ receptor agonists or glucocorticoids to effectively inhibit airway inflammation. However, as the asthma is a chronic disease, long-term application of those drugs may result drug resistance, which resulting certain prognosis impact on patients ${ }^{[3-4]}$. The traditional Chinese medicine treatment is based on dialectical treatment, the conside ration to both the incidental and fundamental of asthma disease can be achieved.

From the perspective of traditional Chinese medicine, asthma is classified into "asthma syndrome" and "asthma syndrome", and its internal cause is considered to have a certain relationship with the dysfunction of kidney, lung and spleen, because the imbalance of Yin and Yang in organs causing the transport disorder of body fluid, which leads to the stagnation of phlegm in the lung. However, most of them are affected by emotional disorder or exogenous evil wind, which leads to the disorder of lung "Qi" (air), causing damages to the spleen and kidney over long time under this situation, and finally resulting the spleen and kidney fail to function properly ${ }^{[5-11]}$. According to relevant studies, the effect of Pingchuan decoction combined with Seretide is significantly better. The results show that there was no significant difference in inflammatory factor index and lung function index between the two groups before treatment. After treatment, the total recovery rate of the experimental group was significantly higher than the control group, the inflammatory factor index and pulmonary function index were improved, $\mathrm{P}<0.05$ with significant difference. This also indicates that Huagai Pingchuan decoction combined with Seretide has better effect, can effectively improve the lung function of patients, reduce inflammatory factors, promote the rapid remission of symptoms of patients, and it is highly safe.

In conclusion, Huagai Pingchuan decoction combined with Seretide has better clinical effect in asthma treatment, which can significantly improve the lung function and inflammatory factor indexes of patients, improved the recovery effect, can therefore widely promote and apply in asthma clinical treatment.

\section{References}

[1] Chang YL, Gao QJ, Xue PN, et al. Clinical efficacy of Sulidil combined with compound isoproterium bromide in the treatment of cough variant asthma $[\mathrm{J}]$. Journal of clinical military medicine, 2018, 46(12): 1473-1475.

To investigate the clinical efficacy of seretide combined with compound ipratropium bromide in the treatment of cough variant asthma [J]. Journal of clinical military medicine, 2018,46 (12): 1473-1475.

[2] Wei F. Clinical efficacy of seretide combined with montelukast in the treatment of cough variant asthma $[\mathrm{J}]$. Journal of contemporary clinical medicine, 2018, 31(2): 3758

[3] Yang KY, Qu XX. Study on the curative effect of Pingchuan Huatan Decoction Combined with acupuncture in the treatment 
of children with asthma in remission [J]. Shaanxi traditional Chinese medicine, 2018, 39 (2): 235-237.

[4] Zheng QZ. Efficacy and safety of Fuzheng Pingchuan decoction combined with conventional western medicine in the treatment of cardiogenic asthma $[\mathrm{J}]$. Clinical research of traditional Chinese medicine, 2018, 10(16): 46-47.

[5] Liu L, Liu R, Chen F. Clinical efficacy of tiotropium bromide combined with Seretide in the treatment of COPD and asth$\mathrm{ma}[\mathrm{J}]$. Internal medicine, 2018, 13 (3): 370-371.

[6] Zhuang C, Shen YY, Liu XM, et al. Clinical observation of Pingchuan recipe combined with Qingre powder acupoint application in the treatment of mild infantile heat asthma [J]. Modern Journal of Integrated Chinese and Western medicine, 2018, 27 (17): 12-15.

[7] Yin XJ. Clinical effect of cetirizine combined with Seretide in the treatment of bronchial asthma $[\mathrm{J}]$. Modern diagnosis and treatment, 2018, 254(2): 91-93.
[8] Yang HT, Chen Z, Song AQ. Clinical efficacy of seretide combined with montelukast in the treatment of acute attack of bronchial asthma and its influence on lung function indexes [J]. Guizhou medical journal, 2018, 42(1): 41-43.

[9] Chen WT, Su KL. Xuanfei Pingchuan decoction in the treatment of infantile asthma and its effect on exhaled nitric oxide [J]. Journal of clinical pathology, 2019(8): 1661-1668.

[10]Wang AL, Yang QZ. Efficacy of Qufeng Pingchuan decoction combined with budesonide in the treatment of children with cough variant asthma and its influence on serum TIMP1, MMP-2 and IL-13 [J]. Global traditional Chinese medicine, 2018 (7): 1116-1119.

[11]Zhao H. Observation on the therapeutic effect of the combination of traditional Chinese medicine for purging lung and asthma, eliminating phlegm and relieving cough in the treatment of cough variant asthma $[\mathrm{J}]$. World latest medical information digest, 2018, 18(32): 139 . 